\title{
Impedance characterization of supported oxygen ion conducting electrolytes
}

\author{
Christian Lenser, ${ }^{* a}$ and Norbert H. Menzler ${ }^{a}$
}

\begin{abstract}
Electrochemical impedance spectroscopy is a widely used tool to study electrochemical reactions in batteries, fuel cells and other electrochemical energy conversion devices. However, conduction processes in the electrolyte of high temperature fuel or electrolysis cells (SOFC / SOEC) are inacessible during operation, severely restricting the information that can be obtained about performance and degradation of the electrolyte. Using the distribution function of relaxation times (DRT), we study the ionic conduction properties and degradation phenomena in multi-layered solid electrolytes, ex situ and at low temperatures. The investigation of full cells in air enables a detailed analysis of the conductivity of supported electrolytes as thin as $1 \mu \mathrm{m}$, as well as the relative contributions of multilayered electrolytes. Furthermore, three case studies are presented concerning the degradation mechanism in SOFC and SOEC operation, showcasing the ability of this technique to distinguish the effects of grain boundary contamination, formation of solid solutions and the formation of porosity on the ionic conductivity of thin, supported electrolytes.
\end{abstract}

Keywords: SOFC; SOEC; Impedance spectroscopy; DRT; electrolyte;

\section{Introduction}

Electrochemical impedance spectroscopy (EIS) is a very popular tool in the field of electrochemistry, $[1,2]$ and is routinely used to investigate the electrochemical properties of electrodes in electrochemical cells. $[3,4]$ In particular, EIS is the method of choice to analyse and model the electrochemical processes in Li-ion batteries,[5-7] or to develop and validate chemo-physical models of electrode reactions for solid oxide cells (SOCS) operated in fuel cells mode (SOFC) or electrolysis mode (SOECS).[8-14] While EIS can be used to evaluate the electrode performance in SOFC single-cell tests in great detail, the information that can be obtained about the electrolyte is very limited. This is due to the fact that at SOFC / SOEC (and also proton-conducting fuel cells, PCFC) operation temperatures $\left(>500{ }^{\circ} \mathrm{C}\right.$ ), the ionic conduction in the electrolyte membrane appears ohmic (i.e. frequency independent) in the frequency regime accessible by EIS, which is typically limited to about $1 \mathrm{MHz}$.

As a consequence, information about the conductivity and degradation phenomena of SOC electrolytes is typically obtained via model experiments on bulk materials and / or post-test analysis of the microstructure.[15-19] Among the degradation phenomena that can be observed for SOECs, increases in ohmic resistance have been observed for electrolyte-supported cells due to materials degradation of Scstabilized zirconia (ScSZ).[19] Significant degradation of the ohmic resistance of fuel electrode-supported cells have been reported by many authors.[17, 20, 21] Since impedance spectroscopy can only provide information about the total ohmic resistance of a test setup and a distinction between different contributions to the ohmic resistance is not possible, the degradation of the electrolyte conductivity cannot usually be attributed to specific processes such as the formation of an insulating grain boundary phase, demixing in the electrolyte or formation of insulating secondary phases. In addition, the ohmic resistance of an SOC during operation is strongly influenced by contact resistance,[22] or parasitic resistances of a test setup. It is therefore necessary to understand the cause for ohmic resistance degradation in great detail in order to develop effective mitigation strategies for such degradation.

In this paper, we present a technique to analyse the ionic conductivity and degradation phenomena in thin, supported electrolytes in great detail. We begin this paper with a brief discussion of the relevant physical mechanism of the impedance characterization of ionic conductors, followed by a description of the technique and data analysis, and subsequently present three different case studies showcasing the potential of this technique.

\subsection{Background}

Using impedance spectroscopy to separate grain and grain boundary contributions to the ionic conductivity of oxide ion conductors is a state-of-the-art method that has been applied to many systems.[23-26] To better understand the frequency dispersion of the current response to an $\mathrm{AC}$ voltage applied to an ionic conductor, we will briefly summarize the interaction between charged particles localized in a potential and an AC electric field. A comprehensive review of the topic is beyond the scope of this paper. The acceleration $a=\frac{d v}{d t}$ induced by an electric field $E$ exerting a force $F$ on a charged particle with mass $m$ is proportional to the strength of the electric field, and the charge of the particle $z$ :

$F=-z e E=m \frac{d v}{d t}$

A charge carrier in an ionic solid that is subjected to a weak electric field of the form $E=E_{0} \sin (\omega t)$ can be discussed as the motion of a damped harmonic oscillator, as discussed by Bisquert, Halpern and Henn.[27] In this model, a charge carrier is bound in a localized potential $U(x)=\frac{k}{2} x^{2}$ and the force exerted by the electric field is balanced by the harmonic potential with a spring constant $k$ and a friction coefficient $\zeta$ : 
$F-k x-\zeta \frac{d x}{d t}=0$

From this ansatz, they derive a frequency $\omega_{\zeta}=k / \zeta=\tau_{\zeta}^{-1}$ above which $\left(\omega>>\omega_{\zeta}\right)$ the ac conductivity is constant, and below which the displacement of the ion by the electric field is slowed down by the restoring force of the harmonic potential. The time constant $\tau_{\zeta}$ is the decay time that the particle needs to return to the equilibrium position. While Bisquert, Halpern and Henn add a relaxation of the electrical potential around the ion to describe long range motion, it becomes clear that above a certain frequency $\omega_{\zeta}$, the ionic charge carriers cannot follow the high frequency of the excitation voltage and the ac impedance becomes constant, while the behaviour below $\omega_{\zeta}$ is governed by the potential around the ion.

To describe the temperature dependence of $\omega_{\zeta}$, we turn to the typical interpretation of the ac impedance of an ionic conductor as a series of resistor-capacitor elements, each with resistance $R$ and capacitance $C$ (RC-elements) and a time constant

$\tau_{R C}=R C$

defining the frequency of maximum loss as $\omega_{\max }=(R C)^{-1}$.[23] Since the capacitance is mainly determined by the geometrical capacitance $C=\epsilon_{0} \epsilon_{r} \frac{A}{d}$ (with $\varepsilon_{0}$ and $\varepsilon_{r}$ the permittivity of free space and the relative permittivity of the material, respectively, $A$ the geometrical area and $d$ the thickness of the sample), it is almost temperature-independent in the absence of a structural phase transition. The temperature dependence of $\omega_{\max }$ can therefore be traced to that of the sample resistance, $\frac{d \omega_{\max }}{d T}=\frac{d R^{-1}}{d T}$. The conductivity of a pure ionic conductor is given by Ohm's law:

$\sigma_{\text {ion }}=|z| n \mu_{\text {ion }}$

where $\sigma_{\text {ion }}$ is the ionic conductivity, $n$ the charge carrier concentration, $z$ the ionic charge, and $\mu_{\text {ion }}$ the ionic mobility. In oxide ion conductors, it is advantageous to analyse ionic conduction in the framework of oxygen vacancies $\left[V_{0}^{* \bullet}\right]$ as the mobile species, yielding:

$\sigma_{\text {ion }}=2 *\left[V_{o}^{\bullet}\right] \mu_{V}$

with $\left[V_{o}^{\bullet}\right]$ being the oxygen vacancy concentration, and $\mu_{V}$ the mobility of the oxygen vacancies. In principle, both $\left[V_{o} \cdot{ }^{\prime}\right]$ and $\mu_{V}$ are temperature activated processes. However, in highly-doped systems such as $\mathrm{Y}_{0.148} \mathrm{Zr}_{0.852} \mathrm{O}_{1.926}$ (8YSZ) or $\mathrm{Gd}_{0.1} \mathrm{Ce}_{0.9} \mathrm{O}_{1.95}(10 \mathrm{GDC}$ ), the oxygen vacancy concentration is determined by the dopant concentration through the electroneutrality condition:

$2^{*}\left[A_{C}^{\prime}\right]=\left[V_{O}^{\bullet}\right]$

where $\left[A_{C}^{\prime}\right]$ denotes the concentration of negatively charged acceptors (such as $\mathrm{Y}^{3+}$ or $\mathrm{Gd}^{3+}$ ) substituting the host cations (such as $\mathrm{Zr}^{4+}$ or $\left.\mathrm{Ce}^{4+}\right)$. In the temperature range relevant for SOC applications $\left(<1000^{\circ} \mathrm{C}\right)$, such high dopant concentrations ensure that $\left[V_{O} \cdot{ }^{*}\right]$ is essentially constant. The temperature dependence of the conductivity is therefore reduced to that of the mobility,

$\mu_{V}=\frac{\mu_{V, 0}}{T} \exp \left(\frac{-H_{m, V}}{k T}\right)$

where $\mu_{v, 0}$ is the pre-exponential term, and $H_{m, v}$ is the migration enthalpy of the oxygen vacancy. We note that while from equation (7), an Arrhenius plot of the conductivity should result in a straight line of In $\sigma \mathrm{T}$ vs $1 / \mathrm{T}$, a slight curvature is often observed experimentally. As was recently expertly discussed by Ahamer et al.,[28] the common interpretation of an additional exponential term describing defect association is highly controversial from a theoretical point of view for non-dilute systems, and the curvature can be explained by multiple available diffusion paths for the oxygen vacancies with different migration barriers as well.

From these considerations, we can conclude that the temperature dependence of the RC time constant $\tau_{R C}=R C=\omega_{\text {max }}^{-1}$ is related to the temperature dependence of the ionic mobility. Next to the differences in the capacitance of grain and grain boundary contributions, the value and temperature dependence of the characteristic frequency $f_{c}=\frac{1}{2 \pi \tau_{R C}}$ can be used to identify conduction processes in a complex ceramic structure, e.g. a bilayer electrolyte consisting of a YSZ ion conductor and a GDC diffusion barrier layer.

A popular technique to improve the frequency resolution of impedance spectroscopy is to calculate the distribution function of relaxation times (DRT). $[29,30]$ The relation between the impedance spectrum $Z(\omega)$ and the DRT $\gamma(\tau)$ is given as:

$Z(\omega)=R_{0}+\int_{0}^{\infty} \frac{g(\tau)}{1+j \omega \tau} d \tau$

where $R_{0}$ is the frequency-invariant (ohmic) resistance of the system and $g(\tau)$ is a suitable function that represents the distribution of relaxation times with $\int_{0}^{\infty} \frac{g(\tau)}{1+j \omega \tau} d \tau=Z_{p o l}(\omega)$, as long as the system fulfils the fundamental criteria of linearity (within the investigated voltage amplitude), causality and time invariance.[30] We note that in this notation, the polarisation resistance $R_{\text {pol }}$ is included in the function $g(\tau)$. We use this notation since it is implemented into the DRTtools program. A slightly different (but synonymous) notation that is often used defines $\int_{0}^{\infty} \frac{g(\tau)}{1+j \omega \tau} d \tau=1$ and writes $\mathrm{R}_{\text {pol }}$ as a factor into equation (9) in front of the integral.[29, 31, 32]

Since impedance data is typically displayed on a semi-logarithmic scale, it is more convenient to write:

$Z(\omega)=R_{0}+\int_{-\infty}^{\infty} \frac{\gamma(\tau)}{1+j \omega \tau} d \ln \tau$

with $\gamma(\tau)=\tau g(\tau)$ and $\int_{-\infty}^{\infty} \frac{\gamma(\tau)}{1+j \omega \tau} d \ln \tau=Z_{\text {pol }}(\omega)$.

The main problem associated to the DRT is that solving the Fredholm integral in equation 9 is an 'ill-posed problem' since there can be many solutions that satisfy the equation. Different approaches to calculate the DRT have been suggested and compared, such as Tikhonov (ridge) regularization,[33, 34], Fourier transform,[31] multi-RQ CNLS fits,[29] or genetic programming.[32, 35] The approach used in this work is the Tikhonov regularization, using Gaussians as discretization functions. The approach of the Tikhonov regularization (as 
implemented in the DRTtools software tool) uses only a single variable, the regularization parameter $\lambda$, to generate the DRT. High values of $\lambda$ have a smoothing effect on the DRT and can obfuscate spectral features, whereas small values of $\lambda$ can lead to oscillatory behaviour in the DRT.

\section{Materials and methods}

Anode-supported SOFCs discussed in this studies were fabricated on tape-cast NiO - 8 mol-\% $\mathrm{Y}_{2} \mathrm{O}_{3}$ doped $\mathrm{ZrO}_{2}\left(\mathrm{Y}_{0.148} \mathrm{Zr}_{0.852} \mathrm{O}_{1.926}, 8 \mathrm{YSZ}\right)$ supports.[36] NiO-8YSZ anode layers, 8YSZ electrolytes, $\mathrm{Gd}_{0.2} \mathrm{Ce}_{0.8} \mathrm{O}_{1.9}$ (GDC20) diffusion barrier layers and $\mathrm{La}_{0.58} \mathrm{Sr}_{0.4} \mathrm{Co}_{0.2} \mathrm{Fe}_{0.8} \mathrm{O}_{3-\delta}(\mathrm{LSCF})$ cathode layers were made via screen-printing. For selected cells (as indicated in the text), thin 8YSZ electrolytes were fabricated by spincoating, and the GDC20 diffusion barrier layers were made by physical vapour deposition (PVD).[37] Cells that had been operated in SOFC / SOEC stacks were removed during post-mortem analysis as fragments.

Impedance spectra in the frequency range of $10^{6}-10^{-1} \mathrm{~Hz}$ were recorded using a Novocontrol spectrometer in a tube furnace in air in the temperature range between $200^{\circ} \mathrm{C}$ and $400^{\circ} \mathrm{C}$. Spectra were recorded during heating and cooling cycles to ensure that the experiment had not led to an appreciable oxidation of the Ni electrode. The spectra were checked for quality with the Kramers-Kronig transformation, using the Lin-KK software tool.[38-40] The surface area of each cell fragment was determined via optical image analysis using the ImageJ software tool.[41] The distribution function of relaxation times (DRT) was calculated using the DRttools software tool, which uses the Tikhonov regularization to calculate the DRT.[34] The choice of regularization parameter $\lambda$ is crucial in this analysis, and depends on the quality of the data. The regularization parameter has been chosen as $\lambda=10^{-2}$ in this work, unless noted otherwise. Note, however, that neither the area nor the center of gravity of a DRT peak is drastically changed by the choice of $\lambda$, as long as there is no overlap with another peak.[33]

The polarization resistance of a certain part of the impedance spectrum can be calculated from the integral of the DRT via $Z_{\text {pol }}=$ $\int_{\tau_{1}}^{\tau_{2}} \frac{\gamma(\tau)}{1+j \omega \tau} d \ln \tau$. For convoluted spectra with several peaks that overlap in their respective $\tau$-range, integration is not straightforward and a fit to the DRT is more convenient. Since we investigate ionic conduction processes exclusively, the corresponding impedance responses are best described by RQ-elements in an equivalent circuit analysis. The DRT of an RQ-element is given by:[29]

$g(\tau)=\frac{1}{2 \pi} \frac{\sin (n \pi)}{\cosh \left(n \ln \tau_{0} / \tau\right)+\cos (n \pi)}$

with $\tau_{0}=\sqrt[n]{R \cdot Y_{0}}$. The admittance of the RQ-element is $Y(\omega)=Y_{0}(j \omega)^{n}$.

Instead of using the above exact function in this paper, we apply a generic fit using Gaussians to the extract information about the area and center of gravity of the relevant peaks in the DRT. The reason for this is that when using a small enough value of $\lambda$ for the transformation so that the typical shape of the RQ-DRT emerges, the DRT shows oscillatory behaviour induced by the small value of $\lambda$. We therefore use larger values of $\lambda$, with the result that the peaks in the DRT assume Gaussian shapes since Gaussians are used as discretization functions. As we show in section 3.1.1, this does not affect the peak area or position in a significant way.

Fitting of the DRT spectra can in principle be done with any fitting software that allows the controlled selection of Gaussian profiles and their numerical fitting. For the present work, we utilized the software tool CasaXPS, which is developed and used for the fitting of X-ray photoelectron spectroscopy data. However, CasaXPS provides essential control of the fitting procedure through restraints on the peak positions and widths, which is important to obtain a fit that corresponds to the physical origin of the DRT. CasaXPS also offers a batchfitting routine and data export opportunity, greatly facilitating the analysis. To control the results obtained through DRT fitting, a conventional software to fit impedance spectra with complex non-linear least squares (CNLS) fitting of an equivalent circuit model was used (Zview) for the reference samples.

Microstructural characterization of ceramics and supported electrolytes was performed using a Hitachi SU 8000 scanning electron microscope (SEM) equipped with a field-emission gun and in-lens detector. The polished ceramics were thermally etched before imaging in order to highlight the grain boundaries. Energy-dispersive X-ray spectroscopy (EDX) was recorded using an acceleration voltage of $20 \mathrm{kV}$ and a solid-state detector.

\section{Results}

Electrochemical characterization of supported electrolytes is challenging due to the co-sintering process necessary to fabricate the thin, supported ceramic membrane. The anode-supported SOFCs used in this work are fabricated using a NiO-YSZ support and anode layer, which only become electrochemically active upon chemical reduction of $\mathrm{NiO}$ to $\mathrm{Ni}$ in reducing atmosphere. As a consequence, it is not possible to use a dense electrode on at least one side of the electrolyte, since the activated $\mathrm{Ni}$ electrode is designed to be porous. However, $\mathrm{Ni}$ is stable against re-oxidation at moderate temperatures, which makes it possible to use the reduced cell in a standard impedance measurement in air with Ni acting as one electrode.[42] The percolating Ni network creates a short-circuit around the YSZ particles in support and anode, which means the reduced full cell is treated as an electrochemical cell Ni|YSZ|GDC|LSCF. 
For a typical impedance experiment, a reversible electrochemical cell is desirable, which means that the electrode reactions are quasilinear with regards to the excitation voltage and do not change during the experiment. For the investigated systems, this condition is not fulfilled with regards to the electrode reactions, since the $\mathrm{Ni}$ electrode is not thermodynamically stable in air and oxidation is mainly hindered kinetically. However, the impedance response of the ceramic electrolyte is separated from the electrode response in the frequency domain, making it possible to reliably analyse the electrolyte.

In the following sections, we will describe the method employed using a well-defined system, a dense 8YSZ ceramic, as an example, followed by the validation of the method on high-performance, bilayer electrolytes. Finally, we will showcase three case studies to outline the utility of this method for the characterization of supported electrolytes related to i) electrolyte degradation during sintering by intermixing with the anode material, ii) electrolyte degradation after 34.000 hours of SOFC operation and iii) identifying the ionic contribution to the ohmic resistance of a high-performance SOFC.

\subsection{Method and data analysis}

Figure 1 a) shows the Nyquist plot of an EIS spectrum recorded on a dense 8YSZ pellet with Pt electrodes in air at $250^{\circ} \mathrm{C}$. The spectrum consists of two well resolved semicircles at high frequencies, as well as the electrode response at lower frequencies. As can be seen in the KK-transform of the data (red triangles) and the residuals of the KK-transformed real and imaginary parts with the measured ones (Figure 1 b)), the impedance response is reliable for the two semicircles representing the grain and grain boundary impedance (between $10^{6} \mathrm{~Hz}$ and $1 \mathrm{~Hz}$ ), while the electrode response is not reliable. While equivalent circuit analysis with 2 RC elements in series is easily feasible with such clearly resolved spectra, the electrode response starts to overlap with the bulk response at higher temperatures, which complicates the analysis. Figure $1 \mathrm{c}$ ) shows the corresponding DRT in the $\tau$-domain. The peaks for grain and grain boundaries are clearly separated, and can easily be distinguished in the $\tau$-domain from the electrode response.

Fitting the DRT spectra using Gaussians yields the area and the time constant describing each peak. Figure 2 a) shows an Arrhenius plot of the temperature dependence of the characteristic frequency $f_{c}=\frac{1}{2 \pi \tau_{R C}}$ for the peaks related to ionic transport across grains and grain boundaries in the dense 8YSZ sample (microstructure is shown in Figure $2 \mathrm{~b}$ )). The activation energy calculated from the slope of the characteristic frequency vs reciprocal temperature is $E_{A}=1.05 \pm 0.005 \mathrm{eV}$ and $E_{A}=1.09 \pm 0.005 \mathrm{eV}$ for the grain and grain boundary contribution, respectively. An analogous evaluation of the resistance yields the same values (within the specified uncertainty), as can be expected from the relation between $f_{\max }$ and $\mu$. The same analysis was done for a dense $\mathrm{Gd}_{0.1} \mathrm{Ce}_{0.9} \mathrm{O}_{1.95}$ ceramic (see Figure $\mathrm{S} 1$ ), yielding $\mathrm{E}_{\mathrm{A}}=$ $0.67 \pm 0.02 \mathrm{eV}$ and $\mathrm{E}_{\mathrm{A}}=0.81 \pm 0.01 \mathrm{eV}$ for the grain and grain boundary, respectively.
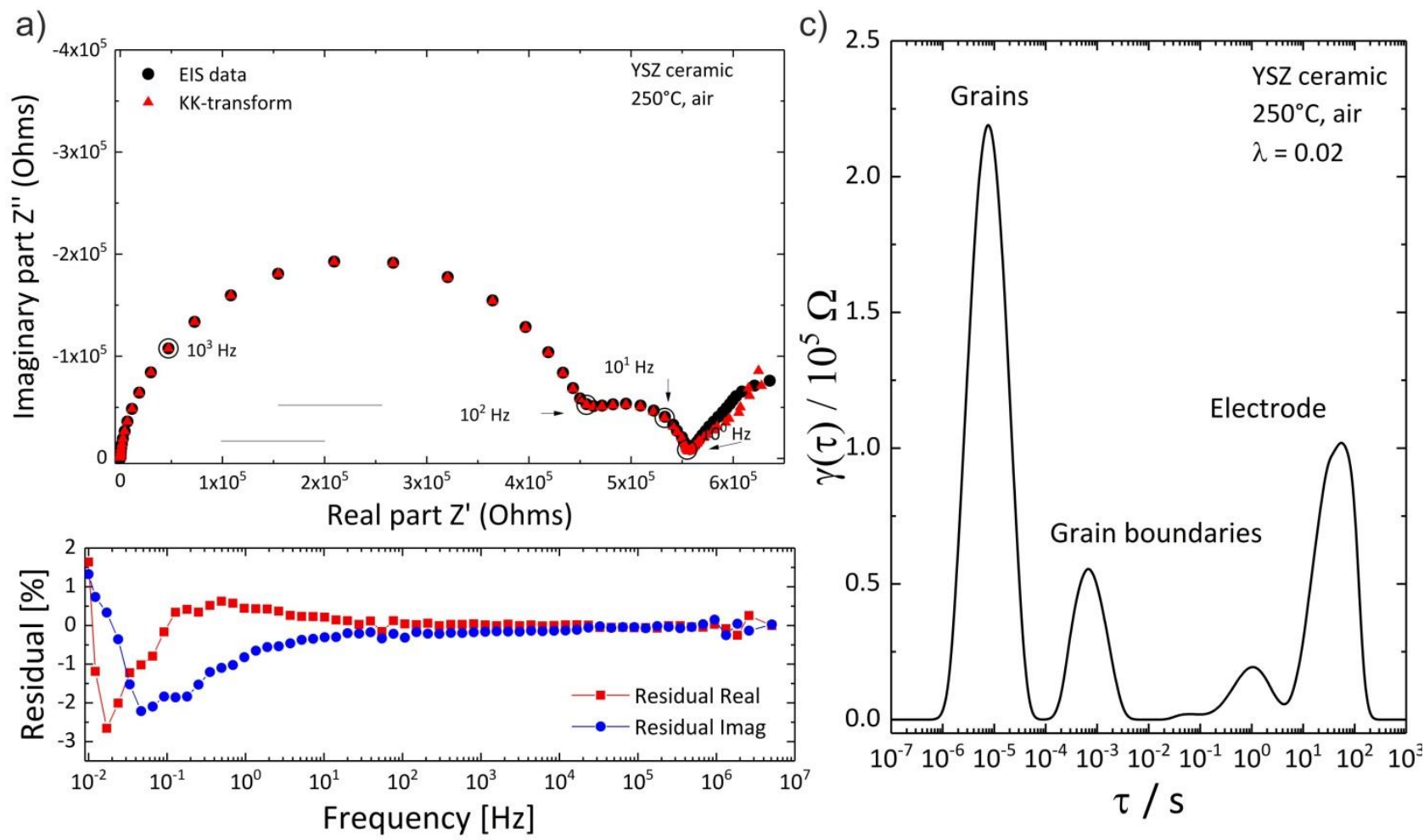

Figure $1 \mathrm{a}$ ): Nyquist plot of the impedance spectrum recorded on a dense $8 \mathrm{YSZ}$ ceramic with Pt electrodes at $250^{\circ} \mathrm{C}$ in air. b) Residuals of the real and imaginary part from the KK-transform, and c) DRT of the spectrum shown in a), with the contributions from grains, grain boundaries and the electrode labelled.

\subsubsection{Error analysis}


In order to estimate the numerical precision of the method, we analyse the calculated impedance response of an artificial RC circuit and compare the fitting results with the true circuit parameters. The analytical DRT of an RC element is a Dirac distribution $\gamma(\tau)=R \cdot \delta(\ln \tau)$, which should yield a single, sharp spike in the DRT. Since the DRT in Figure 3 a) is calculated numerically and with a finite discretization, the shape of the calculated DRT is that of a Gaussian, since the algorithm uses Gaussian discretization functions. Smaller values of $\lambda$ result in a narrower distribution. The objective here is to examine both the effect of the DRT transfer procedure and the fitting of a Gaussian function

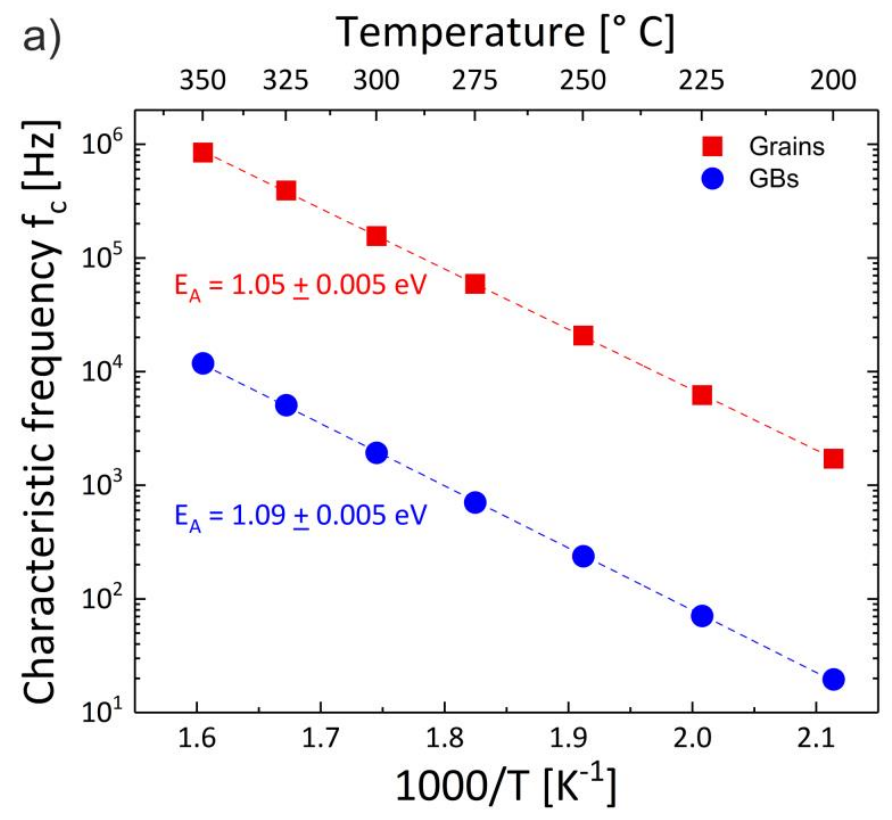

b)

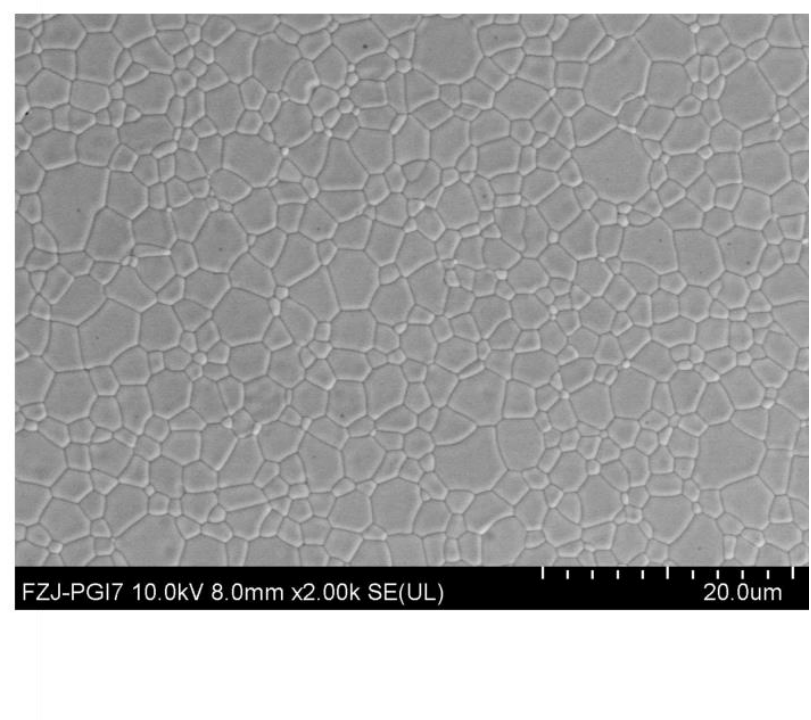

Figure 2 ): Temperature dependence of the characteristic frequency for grain (red squares) and grain boundary (blue circles) impedance, with the activation energy for each process given in the respective color. b) SEM image of the YSZ microstructure after thermal etching.

to the DRT on the precision of the values obtained for the RC circuit.

The black line in Figure 3 a) shows the DRT $\left(\lambda=10^{-2}\right)$ of a simulated RC element consisting of a resistor with $R=100 \Omega$ and a capacitor with $F$ $=10^{-3} \mathrm{~F}$. The corresponding RC time is $\tau_{0}=\mathrm{RC}=0.1 \mathrm{~s}$. The Gaussian fit to the DRT is shown as a dashed red line. From the residual indicated by the dotted blue line, the shape of the DRT is not an ideal Gaussian and the fit shows systematic deviations from the DRT, a result of the Tikhonov regularization used to calculate the DRT.[30] In order to evaluate the precision, Table 1 compares the values for $\mathrm{R}_{\text {, }} \mathrm{C}$ and $\tau_{0}$ obtained through a numerical integration of the DRT (column 'DRT integral') and those obtained from fitting a Gaussian to the calculated DRT. The integrated DRT shows a slight deviation from the reference value for the resistance, and a more significant deviation from the reference value of $\tau_{0}$ (and hence, also for $\mathrm{C}$ ). This deviation is due to the transfer procedure. We note that using a smaller value of $\lambda$ improves the accuracy of the resistance value, but consistently results in the same deviation in the value of $\tau_{0}$.

The Gaussian fit to the DRT slightly increases the inaccuracy in the obtained resistance value, but accurately reproduces the $\tau_{0}$ value of the DRT. We therefore assume these values to be the numerical error resulting from the DRT transform procedure and the consecutive fitting procedure for all values shown in this paper, and the symbol size in each graph is chosen to represent that error bar. Since these are systematic error resulting from the methodology used, these values serve as a rough guideline for the precision we can expect for all values in this paper. We note that the DRT in Figure 3 a) was calculated using a fairly large regularization parameter of $\lambda=10^{-2}$ to be comparable to the data presented in this manuscript and that using smaller values of $\lambda$ does not significantly improve the Gaussian fit to the DRT.
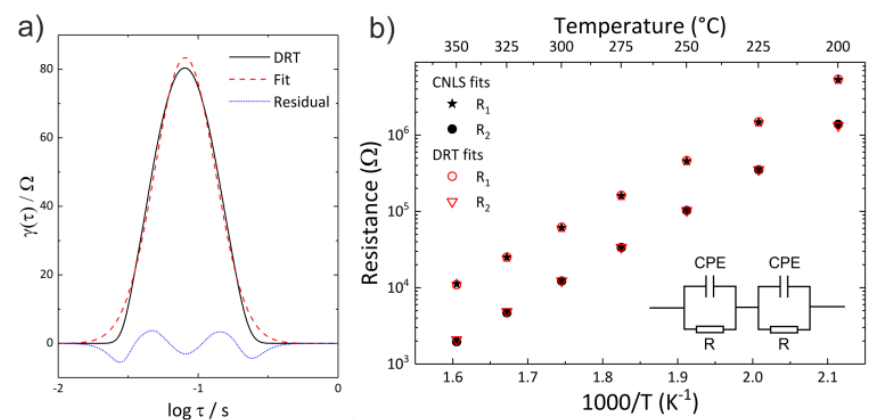

Figure $3 \mathrm{a}$ ): DRT of the impedance response of a model RC circuit (black line), and the fitted Gaussian (dashed red line) as well as the residual of the fit (dotted blue line). b) Comparison of the resistance values for grain $\left(R_{1}\right)$ and grain boundary $\left(R_{2}\right)$ impedance, obtained by CNLS fits (black solid symbols) and DRT fits (red open symbols) of the DRTs obtained from the same data, exemplarily shown in Figure $1 \mathrm{c})$. The equivalent circuit used for the CNLS fit is shown in the inset. 
Table 1: Systematic deviations of the integrated DRT and the Gaussian fit to the DRT for the calculated DRT for a simulated RC element.

\begin{tabular}{|c|c|c|c|c|c|}
\hline Variable & Value & DRT integral & Error & Gaussian Fit & Error \\
\hline $\mathrm{R} 1$ & $100 \Omega$ & $101.41 \Omega$ & $1.41 \%$ & $104.15 \Omega$ & $4.15 \%$ \\
\hline $\mathrm{C} 1$ & $1 \cdot 10^{-3} \mathrm{~F}$ & $8 \cdot 10^{-4} \mathrm{~F}$ & $20 \%$ & $7.8 \cdot 10^{-4} \mathrm{~F}$ & $22 \%$ \\
\hline$\tau_{0}$ & $0.1 \mathrm{~s}$ & $0.081 \mathrm{~s}$ & $19 \%$ & $0.081 \mathrm{~s}$ & $19 \%$ \\
\hline
\end{tabular}

Furthermore, we compare the analysis of the impedance data of the dense 8YSZ ceramic - discussed in section 3.1 - performed with a classical complex non-linear least squares (CNLS) fitting routine of equivalent circuit parameters and the fitting of Gaussians to the respective DRT. A popular way to quantify impedance data is to use a CNLS fit of the parameters of a pre-defined equivalent circuit model to the data. To gain an impression of how the DRT fit compares to the classical CNLS fitting routine, we performed both analyses on the impedance data of a dense 8YSZ ceramic. The advantage of using this simple system is i) that a simple yet physically sound equivalent circuit can be chosen (as shown in the inset of Figure 3 b)), and ii) that the peaks are well separated in the DRT (see Figure 1 b)), excluding any interference from the electrode impedance. Figure $3 \mathrm{~b}$ ) shows a direct comparison of the resistance values obtained by CNLS fits of the impedance data and DRT fits of the DRTs obtained from this data, exemplarily shown in Figure $1 \mathrm{c}$ ). The difference between all fitting values can be seen in Figure S2. The difference for the resistance values is typically smaller than $\pm 3 \%$, and typically smaller than $\pm 6 \%$ for the frequencies (or time constants) and the capacitance. Overall, the agreement is very good and confirms that using Gaussian fits to the DRT can be used to quantify impedance spectra.

\subsection{Validation on supported electrolytes}
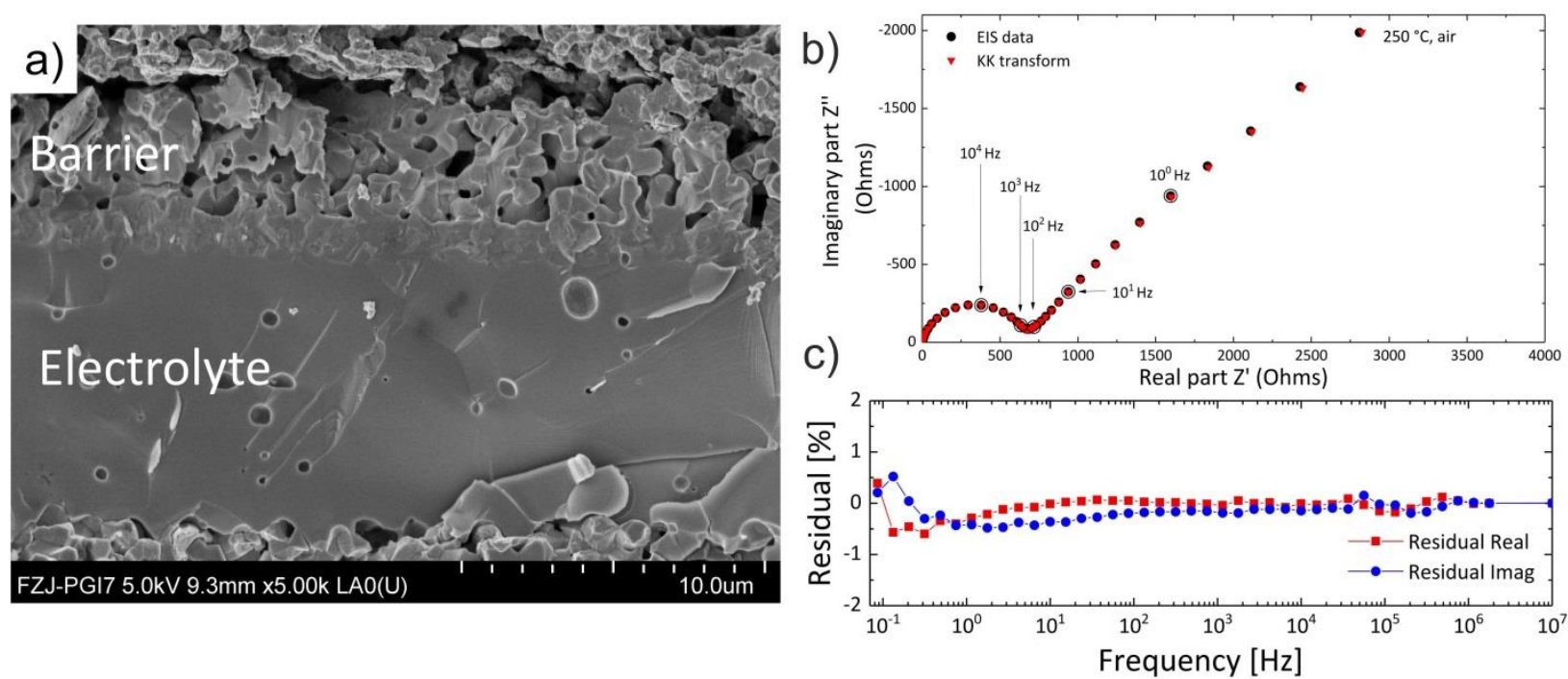

Figure 4 a): SEM cross-section (fracture surface) of a thin, anode-supported bilayer electrolyte. b) Nyquist plot of the impedance data at $250^{\circ} \mathrm{C}$ in air for the microstructure shown in a). c) Residuals from the Lin-KK test for the spectrum in b).

In addition to the difference in activation energy, the characteristic frequencies of grain and grain boundary transport take different values in YSZ and GDC. In this section, we validate whether this criterion is sufficient to distinguish between the grain and grain boundary contributions of both YSZ and GDC in thin, supported electrolytes with different microstructures.

Figure 4 a) shows an SEM micrograph of a fracture surface of an anode-supported fuel cell fabricated by tape-casting of the substrate and screen-printing of all functional layers at Forschungszentrum Jülich. The cell performance was initially tested in dual atmosphere, and subsequently characterized by EIS in air. 
a)

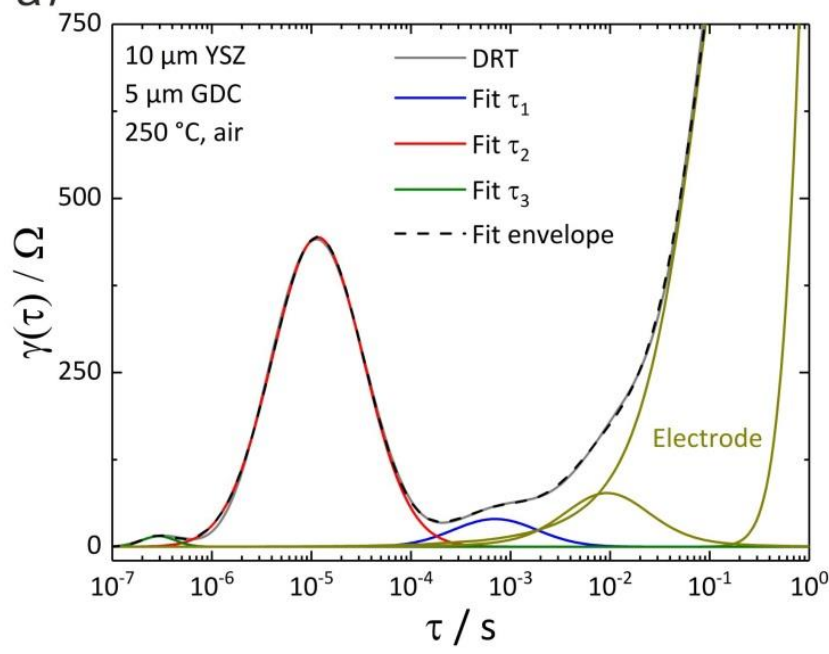

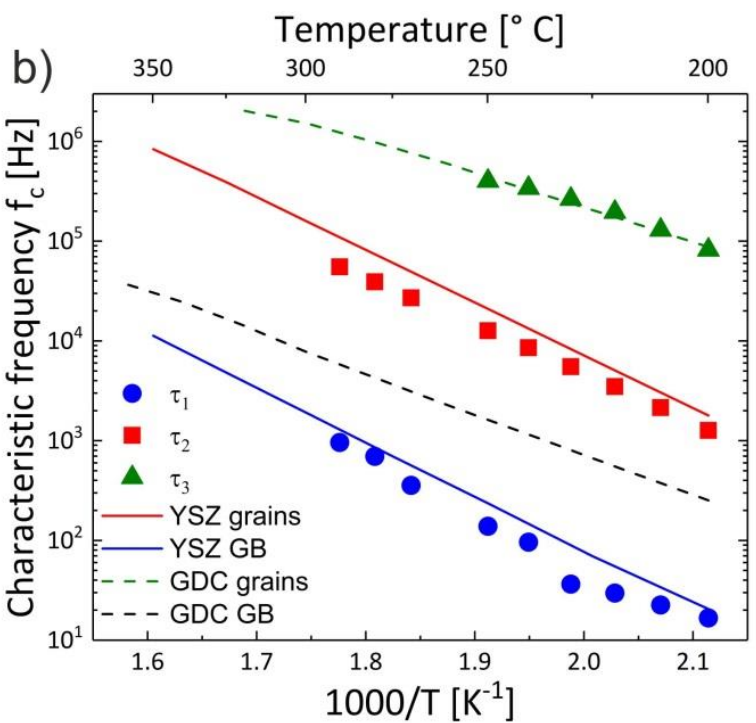

Figure 5 a): DRT of the impedance data at $250^{\circ} \mathrm{C}$ in air, with fits to the respective contributions shown for YSZ grain boundaries ( $\tau_{1}$, blue), YSZ grains ( $\tau_{2}$, red), GDC grains $\left(\tau_{3}\right.$, green) and the electrode components (yellow). b) Characteristic frequency vs. reciprocal temperature for the three components discernible by DRT, along with the reference data from dense YSZ and GDC pellets.

The bilayer electrolyte of this cell consists of a gas-tight, $10 \mu \mathrm{m}$ thick layer of 8YSZ and a $5 \mu \mathrm{m}$ thick porous diffusion barrier layer made from GDC. A representative impedance spectrum is shown in Figure 4 b), recorded at $250^{\circ} \mathrm{C}$ in air, is dominated by the electrode response at low frequencies, and clearly shows several contributions at high frequencies as well. The residuals of the linear KK test are shown in Figure $4 \mathrm{c}$ ), indicating that the impedance data is reliable over the whole frequency range. Using equivalent circuit analysis is complicated in this scenario, since the frequency overlap between electrode and electrolyte response creates a correlation between the accuracy of the model of the electrode response and the electrolyte response, which is undesirable for the analysis of the electrolyte due to the complex models used to describe the asymmetrical electrodes.[12] In contrast, the DRT shown in Figure 5 a) shows clearly resolved peaks in the high frequency range, although a certain overlap exists between the electrode response and the electrolyte response as well. However, the peak fitting procedure for DRT can be performed independent of a physical model for the electrode, since the only objective for the fit shown in Figure 5 a) is an accurate reproduction of the spectrum. In this manner, the DRT spectrum can be labeled with three peaks (labeled $\tau_{1}, \tau_{2}$ and $\tau_{3}$ ) for the electrolyte and four peaks for the electrode. To identify the respective contributions to the DRT, the time constants $\tau$ are converted into characteristic frequencies $f_{\max }$ and compared to the characteristic frequencies of dense YSZ and GDC ceramics. Figure 5 b) shows the latter as solid and dashed lines (YSZ and GDC, respectively) for grains (red and green) and grain boundaries (blue and black). The main contributions to the electrolyte impedance $\left(\tau_{1}\right.$ and $\tau_{2}$ ) can be identified as the grain (red squares, $\left.\tau_{2}\right)$ and grain boundary (blue circles, $\tau_{1}$ ) impedance of the 8YSZ layer due their excellent agreement with the magnitude and temperature dependence of the characteristic frequencies for 8YSZ. In addition, $\tau_{3}$ (green triangles) can be identified as the grain impedance of the GDC layer, which vanishes above a temperature of $250^{\circ} \mathrm{C}$ since the contribution to the impedance becomes too small to quantify. The characteristic
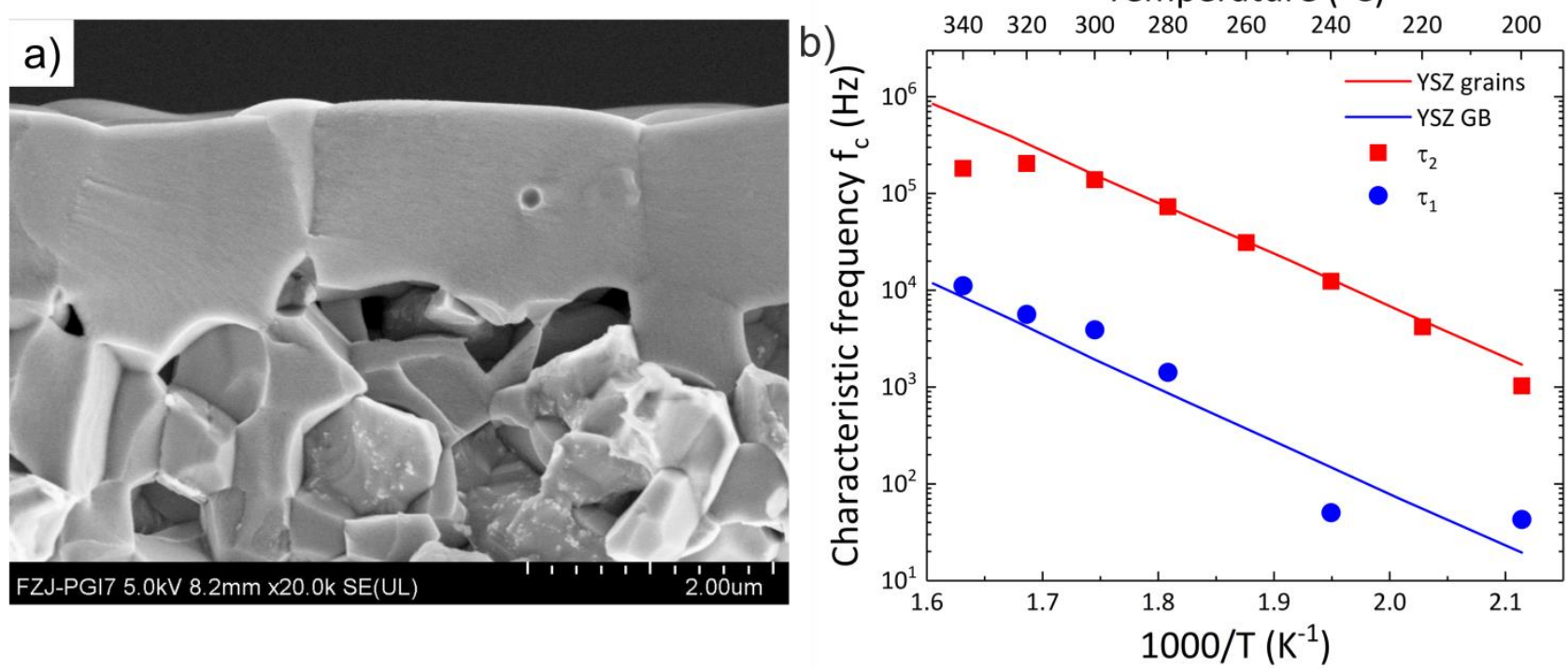

Figure 6 a): SEM cross-section (fracture surface) of a thin, anode-supported 8 YSZ electrolyte. b) Characteristic frequencies vs. reciprocal temperature for the microstructure shown in a). 
frequency of the GDC grain boundary impedance is located between those of the YSZ grain and grain boundary impedance, as shown in Figure $5 \mathrm{~b}$ ). It is therefore likely that the peak related to the GDC grain boundary impedance cannot be observed in the DRT due to significant overlap with the two much higher peaks related to the YSZ electrolyte.

Further validation of this method is obtained from the examination of a thin 8YSZ electrolyte fabricated by successive spin-coating of a nanoparticle dispersion and an alkoxide-based sol,[43] with an average thickness of $1.4 \mu \mathrm{m}$. The size of the 8YSZ grains in the electrolyte exceeds the layer thickness as shown in Figure 6 a), so that it was postulated that the impedance of the electrolyte is dominated by the grains.[44] For impedance testing, a dense Pt electrode was deposited by magnetron-sputtering and subsequently, Ag-paste was applied to the dense Pt layer to improve electrical contact. The characteristic frequencies obtained by fitting of the DRT are plotted vs. reciprocal temperature in Figure 6 b). Reference data from a dense 8YSZ ceramic is shown as a solid

red line for the 8YSZ grains and a solid blue line for the 8YSZ grain boundaries. The magnitude and temperature dependence of the characteristic frequency reveals that peak $\tau_{2}$ is associated with the grain impedance of the 8YSZ electrolyte (red squares). However, an additional peak $\tau_{3}$ can be discerned in the DRT, which is assigned to 8YSZ grain boundaries (blue circles). This peak overlaps significantly with the electrode response at temperatures below $280^{\circ} \mathrm{C}$, so that the data points at the lowest temperature should be treated as inaccurate.

The appearance of the grain boundary contribution is somewhat unexpected, given the fact that in the brickwork model, only grain boundaries orthogonal to the current path are expected to contribute to the impedance since grain boundaries parallel to the current path
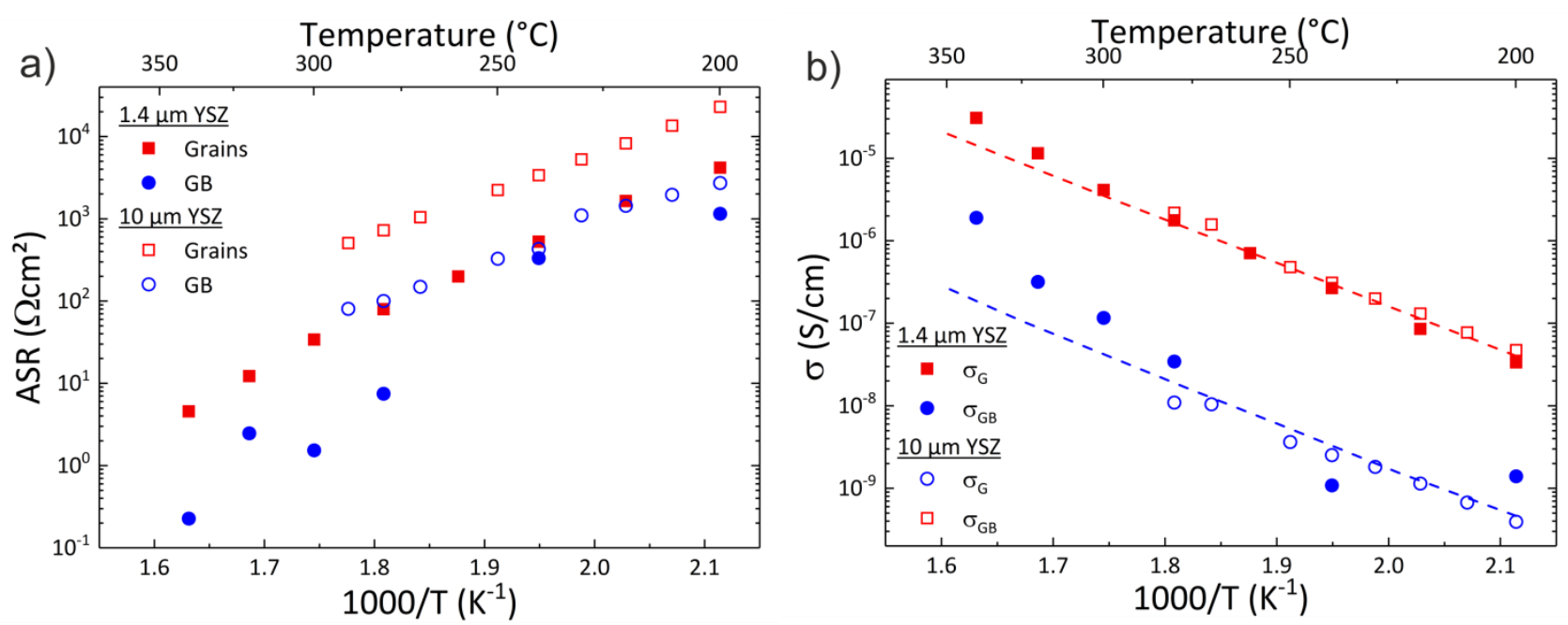

Figure 7 a): ASR values determined by DRT fits for two thin supported 8YSZ electrolytes. b) Comparison of the calculated grain (red squares) and grain boundary (blue circles) conductivity for the $1.4 \mu \mathrm{m}$ electrolyte (solid symbols) and the $10 \mu \mathrm{m}$ electrolyte (open symbols), as well as the respective conductivities of a dense $8 Y S Z$ ceramic (dashed lines).

are shortened by the grains. There are two possible interpretations for this component: i) the Ni electrode may not be active directly at the electrolyte, and so the grain boundary component may derive from the Ni-YSZ electrode structure, or ii) the interfaces to Ni and Pt have similar properties to grain boundaries. However, it is clearly possible to distinguish between grain and grain boundary impedance even for $1.4 \mu \mathrm{m}$ thin electrolytes.

In order to showcase the potential for the comparison of supported electrolyte performance, Figure 7 a) shows the grain and grain boundary ASR values determined for the 8YSZ electrolyte consisting of a $10 \mu \mathrm{m}$ YSZ layer (shown in Figure 4 a)) and a $1.4 \mu \mathrm{m}$ YSZ layer (shown in Figure 6 a)). While the ASR of both electrolytes is dominated by the grain conductivity, the grain ASR of the thin electrolyte layer is only as large as the grain boundary ASR of the thick layer. Using the known electrolyte thickness, we calculate the conductivity values for the 8YSZ grains in both electrolytes (shown in Figure 7 b)) and find them to agree very well with each other and also with the grain conductivity determined on a dense 8YSZ ceramic (red dashed line). In order to calculate the grain boundary conductivity, we use a correction factor to the geometry that is based on the capacitance ratio of grain boundary to grains $C_{g} / C_{g b}=l_{g b} / l_{g}$, where $\mathrm{C}$ and I are the capacitance and physical dimension, respectively, and the index $g$ and $g b$ stand for grain and grain boundary, respectively. The agreement of the grain boundary conductivity with the bulk is also very good for the $10 \mu \mathrm{m}$ electrolyte, and reasonable for the $1.4 \mu \mathrm{m}$ electrolyte, given the inaccuracy of the grain boundary conductivity determined for the $1.4 \mu \mathrm{m}$ electrolyte at high temperatures. This analysis shows that specific conductivity values equal to the bulk conductivity of the material can be extracted from the impedance data, which is an important prerequisite for the analysis of degradation phenomena of thin, supported electrolytes by DRT fitting.

We note that a similar analysis has been done on thin, supported electrolytes using micro-electrodes contacted by tungsten tips by Shin et al, who arrive at similar (yet somewhat less detailed) conclusions. [45] When compared to the present work, the main drawback seems to be the influence of the tungsten tips on the temperature distribution in the microelectrodes setup, which is discussed as a source of error by Shin et al. and has been described in detail by Huber et al. for microelectrode setups. [46]

\subsection{Case studies for electrolyte degradation in ASCs}



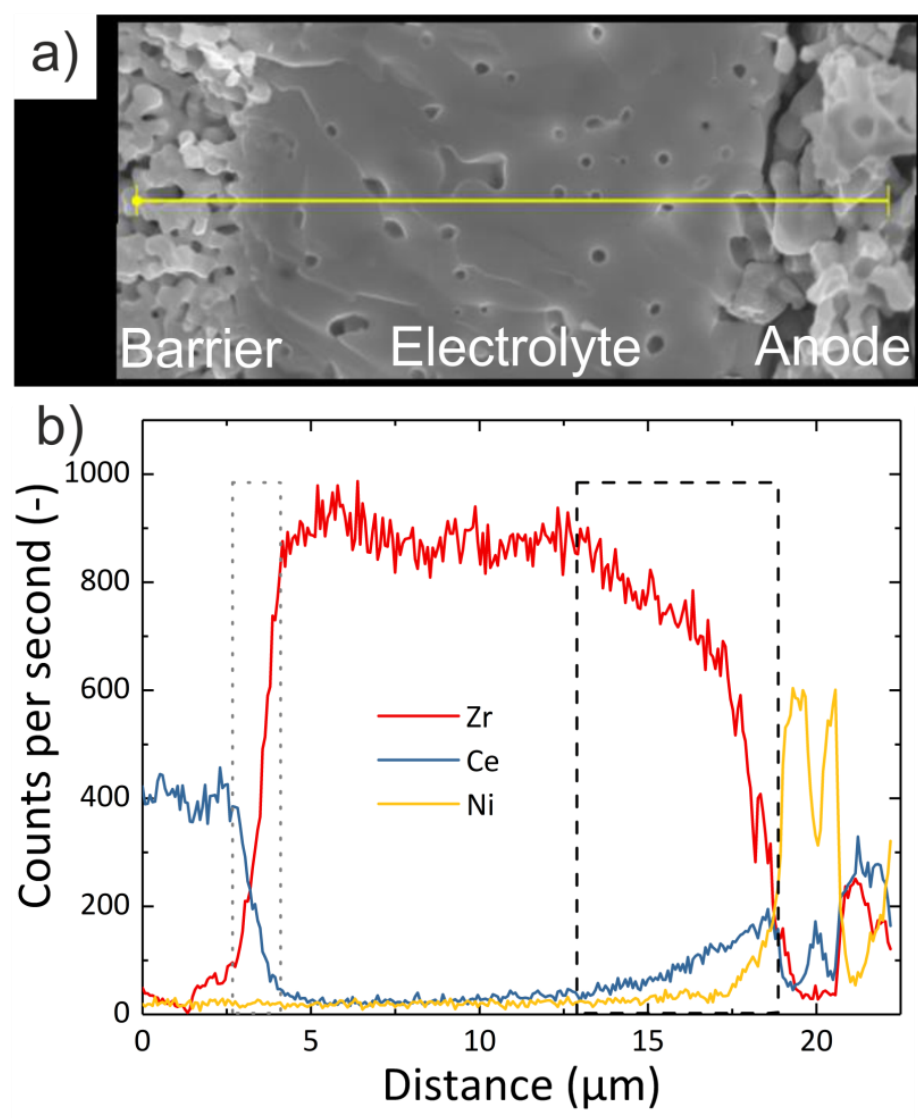

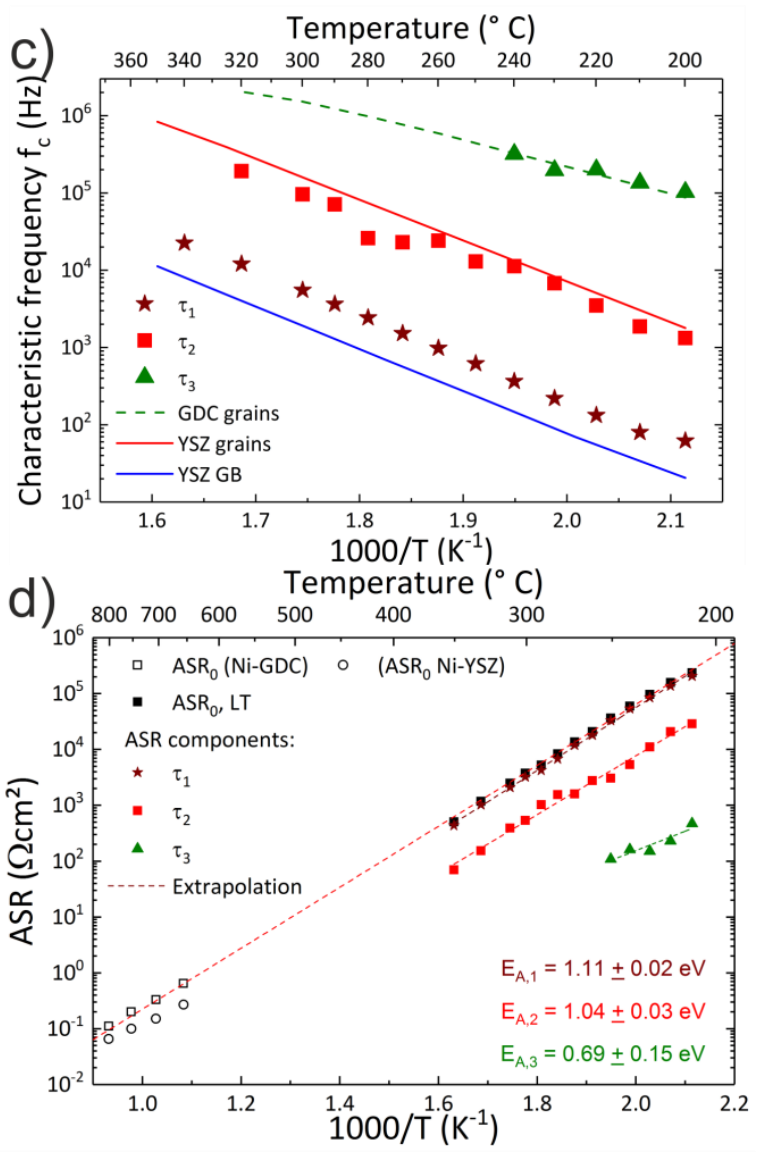

Figure 8 a): SEM cross-section of the fractured cell with Ni-GDC anode after testing. The yellow line indicates the location of the EDX line scan. b) X-ray emission intensity of the main constituents for the line indicated in a). The two interdiffusion zones at air and fuel side of the electrolyte are marked by a gray, dotted box and a black, dashed box, respectively. c) Characteristic frequency vs. reciprocal temperature for the three peaks observed in the DRT ( $\tau_{1}, \tau_{2}$ and $\left.\tau_{3}\right)$, along with the reference data for GDC grains (dashed green line), YSZ grains (solid red line) and YSZ grain boundaries (solid blue line). d) Temperature dependence of the area specific resistance (ASR) corresponding to each of the three DRT peaks. Activation energies are indicated for each component.

to the ionic conductivity of the electrolyte.

\subsubsection{Interdiffusion between YSZ and GDC during sintering}

Replacing 8YSZ with GDC as the ionic conductor in Ni-based cermets has been shown to improve both the electrochemical performance as well as the resistance against sulfur- and carbon poisoning. $[47,48]$ We recently demonstrated that integrating a NiO-GDC anode into the standard fabrication route of an anode-supported fuel cell with 8YSZ electrolyte leads to a significant loss (> 50\%) of performance in cell tests, and explored possible explanations due to interdiffusion between the GDC in the anode and the 8YSZ electrolyte, mainly measured on powder mixtures.[49] Here, we apply impedance analysis of the ionic conductivity of the actual cell to quantify the effect of the interdiffusion on the ionic conductivity of the electrolyte.

Figure 8 a) shows a SEM cross-section of the fractured cell after testing, with a yellow line indicating the location of the EDX line scan shown in Figure $8 \mathrm{~b}$ ). The line scan runs from the GDC diffusion barrier layer through the electrolyte and into the Ni-GDC anode. Since the sample is fractured, quantification of the EDX spectra is not reliable due to possible intensity fluctuation induced by the rough surface. In addition, the spatial resolution in EDX line scans suffers from the large interaction volume of the scattered electrons in the solid. However, an impression of the spatial resolution in this particular experiment can be gleaned from the abrupt intensity changes in the anode layer between $\mathrm{Ni}$ and the oxide phases, showing that the EDX scan can resolve chemically different materials with a resolution better than 300 $\mathrm{nm}$ in a qualitative manner.

A comparison of the two interfaces of the electrolyte is very instructive to determine the amount of interdiffusion during cell manufacturing. The interface to the GDC barrier layer on the air side of the cell is formed during a sintering step at $1300{ }^{\circ} \mathrm{C}$, and a small interdiffusion zone (approx. $1.5 \mu \mathrm{m}$ ) can be observed via EDX (indicated by a dotted gray box in Figure 8 b)). In contrast, the interdiffusion zone at the interface to the GDC anode is approximately $6 \mu \mathrm{m}$ thick (dashed black box in Figure 8 b)), and a substantial amount of Ce can be found in the electrolyte, which can be explained by the high co-sintering temperature during cell manufacturing $\left(1400{ }^{\circ} \mathrm{C}\right)$ and the 

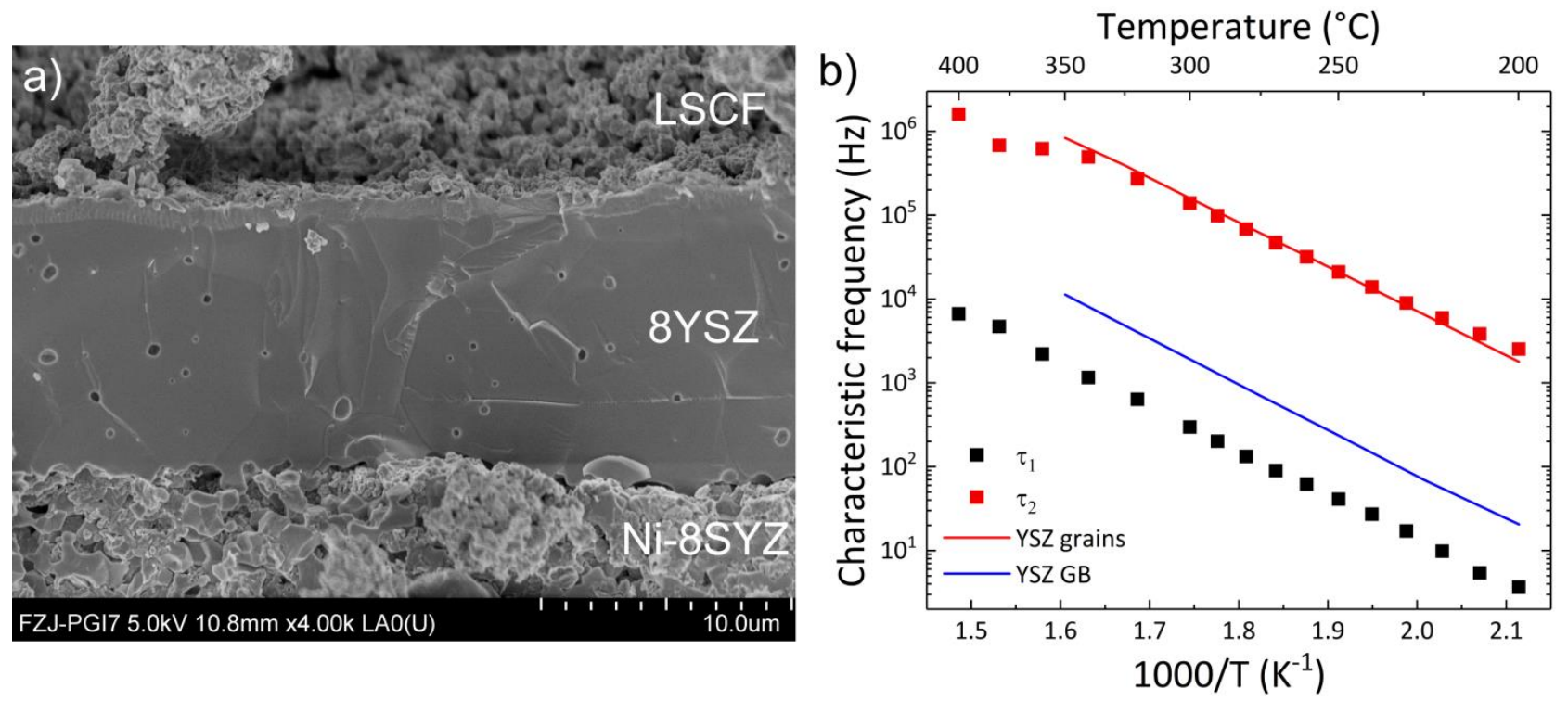

Figure 9 a): SEM cross-section of a fracture surface of the degraded cell after 34.000 hours of operation. b) Characteristic frequency vs. reciprocal temperature for the peaks observed in the DRT ( $\tau_{1}$ and $\tau_{2}$ ), along with the reference data for YSZ grains (solid red line) and YSZ grain boundaries (solid blue line).

presence of $\mathrm{NiO}$ in the anode, which has been shown to accelerate the interdiffusion of 8YSZ and GDC. [49] This amount of interdiffusion can be assumed to decrease the ionic conductivity of the electrolyte.[49-51]

In order to quantify the decrease of ionic conductivity and the mechanism behind it, we performed impedance analysis on the cell after it had been tested at SOFC temperatures. The characteristic frequencies of the three distinguishable components $\tau_{1}, \tau_{2}$ and $\tau_{3}$ in the DRT are shown in Figure $8 \mathrm{c}$ ). Comparing the characteristic frequencies of these components with the reference materials, $\tau_{3}$ can be assigned to the grain impedance of the GDC barrier layer, and $\tau_{2}$ can be assigned to the grain conductivity of the 8YSZ electrolyte. Component $\tau_{1}$ does not correspond to a characteristic frequency of either the 8YSZ or GDC reference. However, the characteristic frequency is closest to that of the YSZ grain boundaries. In order to establish the physical origin of $\tau_{1}$, we can extract the activation energy and the specific capacitance from the impedance data.

Analysis of the activation energy (listed in Table 2) of each of the components yields values for $\tau_{2}$ and $\tau_{3}$ that are in excellent agreement with the reference value established in this temperature interval for the 8YSZ and GDC grains, respectively, but the activation energy for $\tau_{1}$ is slightly higher than expected for 8YSZ grain boundaries. However, it is in excellent agreement with the activation energy determined for ionic transport in $\mathrm{Gd}_{0.086} \mathrm{Ce}_{0.346} \mathrm{Y}_{0.073} \mathrm{Zr}_{0.494} \mathrm{O}_{1.92}$, [49] and other bulk zirconia-ceria mixtures. [50, 51]

In typical microstructures of highly doped ionic conductors, the grain size is usually in the regime of $10^{-6} \mathrm{~m}$, whereas the width of the grain boundaries in highly doped oxide ion conductors is typically on the order of $10^{-9} \mathrm{~m} .[23,52,53]$ The difference in the capacitances of grain and grain boundary impedance reflects the large difference in the respective physical extension, and is therefore an excellent tool to determine whether an unknown contribution to the impedance is related to grains or grain boundaries. Therefore, the most important tool to clarify the nature of component $\tau_{1}$ is the analysis of the specific capacitance through the RC time given in equation (3) ( $\tau=R C$ ). For comparison between different samples, it is instructive to calculate not just the capacitance $C=\frac{\tau}{R}$, but also the dielectric constant

$\varepsilon=C \frac{l}{A}=\frac{\tau \cdot l}{R \cdot A}$

since the measured capacitance depends on the specific geometry and the dielectric constant does not. We can calculate $\varepsilon$ using the geometry of the different layers of the electrolyte (and also the ceramic reference samples) where $I$ and $A$ are the thickness and measured area of each layer. As described above, the assumed value of / for the grain boundaries is typically 3 orders of magnitude too large, leading to a much lower value for $\varepsilon$ when a grain boundary process is analysed. Based on the SEM analysis, the values of $I$ are chosen as $4 \mu \mathrm{m}$ for the GDC barrier layer $\left(\tau_{3}\right), 9 \mu \mathrm{m}$ for the YSZ electrolyte without Ce-interdiffusion $\left(\tau_{2}\right)$ and $6 \mu \mathrm{m}$ for the interdiffusion zone between anode and electrolyte $\left(\tau_{1}\right)$. From the calculated values of the $\varepsilon$ given in Table 2 we see that component $\tau_{1}$ is related to a grain impedance. From the ASR values shown in Figure $8 \mathrm{~d}$ ), the known doping concentrations of 8YSZ and GDC (see Table 2) and equations (5) and (7), it is possible to

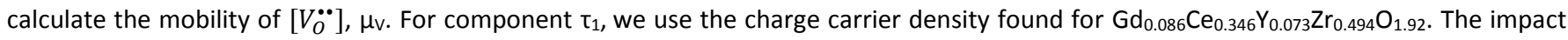
of the likely inaccuracy of that assumption on the mobility is actually very small, since all components in the electrolyte have a high oxygen vacancy concentration that only deviates by less than a factor of 2 . We note further that there are not enough data points for a reliable evaluation of $\mu_{\mathrm{v}}$ related to component $\tau_{3}$. The mobility value at $200^{\circ} \mathrm{C}$ for component $\tau_{2}$ agrees well with that of the YSZ grains, but the mobility value of $\tau_{1}$ is an order of magnitude smaller, which was also previously observed for $\mathrm{Gd}_{0.086} \mathrm{Ce}_{0.346} \mathrm{Y}_{0.073} \mathrm{Zr}_{0.494} \mathrm{O}_{1.92}$. [49]

To summarize the analysis of the electrolyte impedance of an 8YSZ electrolyte co-sintered with a NiO-GDC anode layer, we find an impedance contribution which is not associated to either GDC or 8YSZ. 
Table 2: Activation energy $E_{A}$ (calculated from ASR values) and specific capacitance $C$ at $200^{\circ} \mathrm{C}$ for $\tau_{1}, \tau_{2}, \tau_{3}$ and the reference materials

\begin{tabular}{|l|c|c|c|c|c|c|c|}
\hline & $\tau_{1}$ & $\tau_{2}$ & $\tau_{3}$ & YSZ grains & YSZ GB & GDC grains & GDC GB \\
\hline $\mathrm{E}_{\mathrm{A}}[\mathrm{eV}]$ & $1.11 \pm 0.02$ & $1.04 \pm 0.03$ & $0.69 \pm 0.15$ & $1.05 \pm 0.005$ & $1.09 \pm 0.005$ & $0.67 \pm 0.02$ & $0.81 \pm 0.01$ \\
\hline $\begin{array}{l}\varepsilon[\mathrm{F} / \mathrm{cm}] \\
200^{\circ} \mathrm{C}\end{array}$ & $7.5 \cdot 10^{-12}$ & $3.7 \cdot 10^{-12}$ & $1.3 \cdot 10^{-12}$ & $1.8 \cdot 10^{-12}$ & $5.8 \cdot 10^{-9}$ & $3.5 \cdot 10^{-12}$ & $1.7 \cdot 10^{-9}$ \\
\hline $\mathrm{I}[\mu \mathrm{m}]$ & 6 & 9 & 4 & - & - & - & - \\
\hline$\left[V_{O}^{\bullet-}\right][49]\left[\mathrm{cm}^{-3}\right]$ & $2.22 \cdot 10^{21}$ & $2.18 \cdot 10^{21}$ & - & $2.22 \cdot 10^{21}$ & & $1.26 \cdot 10^{21}$ & \\
\hline $\begin{array}{l}\mu_{\mathrm{V}} \text { at }\left(200^{\circ} \mathrm{C}\right) \\
\mathrm{cm}^{2} / \mathrm{Vs}\end{array}$ & $6.8 \cdot 10^{-12}$ & $8.4 \cdot 10^{-11}$ & - & $6.8 \cdot 10^{-11}$ & - & $1.3 \cdot 10^{-9}$ & - \\
\hline
\end{tabular}

From the capacitance values, we can determine this impedance to be related to a transport process in the grains, and not in the grain boundaries. The activation energy of ionic conductivity as well as the mobility of the ionic charge carriers show excellent agreement with values previously found for a mixed oxide obtained by interdiffusion of 8YSZ and 20GDC. Combining these findings, we can confidently say that the additional contribution to the impedance $\tau_{1}$ arises from the diffusion of Ce into the 8YSZ electrolyte, and the corresponding decrease in ionic conductivity. The ASR values extracted from the DRT fit in Figure $8 \mathrm{~d}$ ) show clearly that in this temperature interval, the impedance of this YSZ-GDC mixed phase dominates the overall impedance response.

Having determined the physical origin of the additional impedance in the temperature interval between $200{ }^{\circ} \mathrm{C}$ and $340{ }^{\circ} \mathrm{C}$, the question arises whether this impedance is relevant at SOFC operation temperature. From the temperature dependence of component $\tau_{1}$ given in Figure $8 \mathrm{c}$ ), it is easily determined that the characteristic frequencies at $700{ }^{\circ} \mathrm{C}$ will not be accessible by the usual impedance spectroscopy techniques, which are instrumentally limited to about $1 \mathrm{MHz}$, and will appear ohmic in nature. Figure $8 \mathrm{~d}$ ) shows the measured ohmic ASR values of the cell with $\mathrm{Ni}-\mathrm{GDC}$ anode (open, black squares) at a temperature between $800{ }^{\circ} \mathrm{C}$ and $650{ }^{\circ} \mathrm{C}$, as well as the total ASR determined from the electrolyte impedance between $340{ }^{\circ} \mathrm{C}$ and $200{ }^{\circ} \mathrm{C}$ (solid black squares). When the low-temperature impedance data

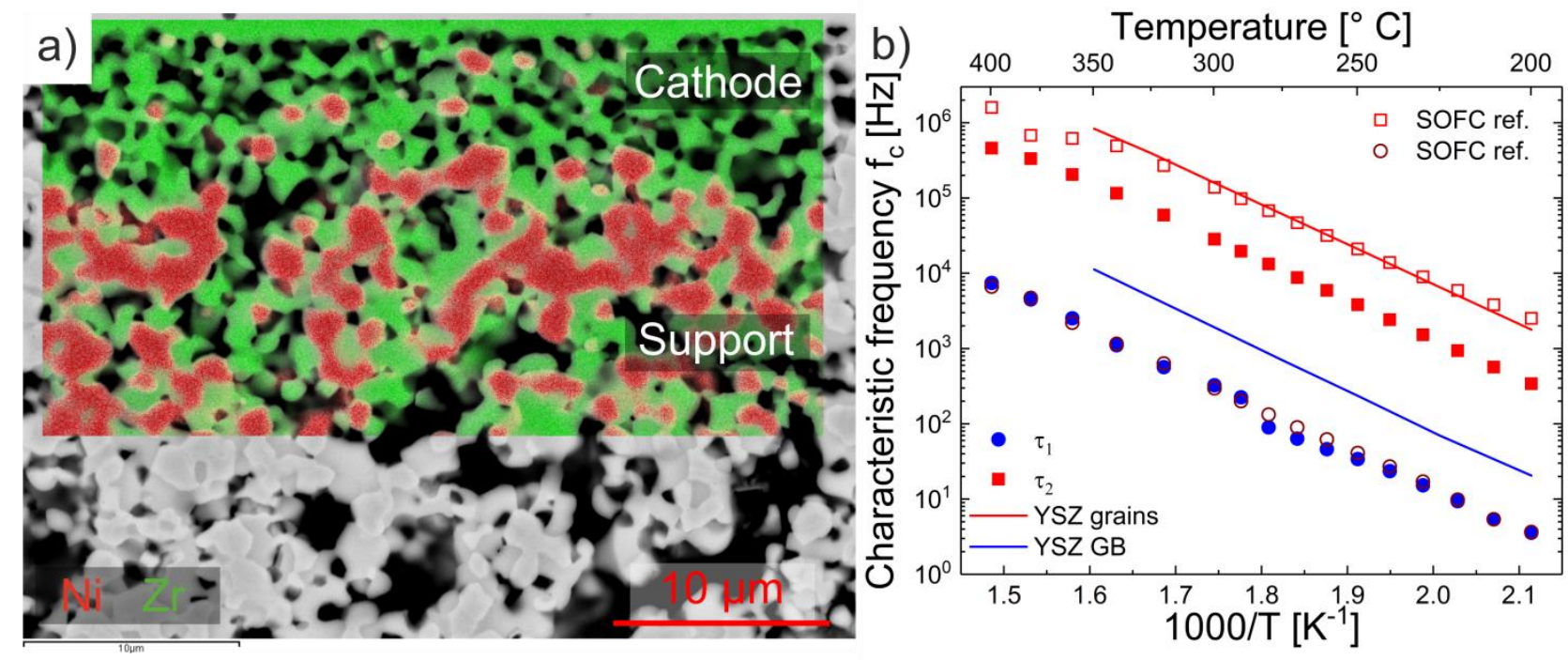

Figure 10 a) SEM cross-section (with superimposed EDX-mapping) of an SOEC cell after 20,000 hours of operation. Ni is shown in red, $\mathrm{Zr}$ in green. b) Characteristic frequency vs. reciprocal temperature for the peaks observed in the DRT ( $\tau_{1}$ and $\tau_{2}$ ), along with the reference data for YSZ grains (solid red line) and YSZ grain boundaries (solid blue line). The characteristic frequencies of the SOFC cell shown in 3.3 .2 are shown for comparison as red, hollow squares (YSZ grains) and brown, hollow circles (YSZ grain boundaries).

is extrapolated to SOFC operation temperatures (dashed, red line), we see that there is excellent agreement with the measured data. For comparison, the ohmic ASR of an otherwise identical cell with a Ni-YSZ anode is shown (open black circles), which is clearly lower.

\subsubsection{Electrolyte degradation during long-term SOFC operation}

The post-mortem analysis of an SOFC stack operated for more than 34,000 hours at $700^{\circ} \mathrm{C}$ at Forschungszentrum Jülich recently found progressive degradation in one of the four planes of the stack, which could be traced back to catastrophic failure of the electrolyte through diffusion of $\mathrm{Mn}$-ions along the grain boundaries of the 8YSZ electrolyte.[54] Here, we apply impedance analysis to a part of the same progressively degraded cell to examine whether we can isolate the effect of $\mathrm{Mn}$ on the grain boundaries on the electrolyte conductivity. We note that the examined piece of the cell did not show the catastrophic failure observed in other places, as shown in Figure 9 a). The electrolyte consists of a $8 \mu \mathrm{m}$ thin YSZ layer and a $0.5 \mu \mathrm{m}$ thin, dense GDC layer. 
The characteristic frequencies of the DRT components corresponding to the electrolyte are shown in Figure 9 b), along with the reference frequencies for YSZ ceramics. Unlike the screen-printed GDC layer in 3.3.1, the contribution of the 0.5 $\mu \mathrm{m}$ GDC layer (deposited by PVD) to the cell impedance is too small to measure reliably. It is clear from this comparison that $\tau_{2}$ corresponds to the grain impedance of the YSZ electrolyte, and the dielectric constant calculated from equation (11) yields a value between $2-5 \cdot 10^{-12} \mathrm{~F} / \mathrm{cm}$ across the measured temperature range, consistent with grain conduction. However, the characteristic frequency calculated from $\tau_{1}$ is significantly smaller than that expected for the grain boundary contribution of 8YSZ. The calculated dielectric constant $\varepsilon$ of component $\tau_{1}$ (using a layer thickness of 8 $\mu \mathrm{m}$ ) yields a value of roughly $2-4 \cdot 10^{-9} \mathrm{~F} / \mathrm{cm}$ in the temperature range between $200^{\circ} \mathrm{C}$ and $400{ }^{\circ} \mathrm{C}$, confirming that it is indeed related to a grain boundary process. Note that this calculation still assumes a negligible difference in the relative permittivity of the grains and grain boundaries, which is likely not the case when the grain boundary is contaminated by Mn ions. However, any changes in the relative permittivity cannot be expected to be responsible for the three orders of magnitude difference in the dielectric constant between components $\tau_{1}$ and $\tau_{2}$, supporting the conclusion of a grain boundary phase.

In turn, the conductivity of this grain boundary phase is approximately one order of magnitude lower than that of the "pure" 8YSZ grain boundaries of the reference cell described in section 3.2. Correspondingly, the contribution of the grain boundaries to the electrolyte resistance is enhanced. However, the resistance of the grains remains the largest contribution to the ASR, which means that Mn-diffusion along the grain boundaries in 8YSZ electrolytes has a negligible impact on cell performance until it leads to catastrophic failure of the electrolyte.

\subsubsection{Cell degradation during long-term SOEC operation}

The long-term operation of solid oxide electrolysis cells (SOEC) has been repeatedly shown to lead to a degradation phenomenon not observed in SOFC operation: the depletion of $\mathrm{Ni}$ in the electrochemically active zone of the fuel electrode.[20, 21, 55, 56] Although a hypothesis about a possible mechanism based on $\mathrm{Ni}(\mathrm{OH})_{2}$ gas-phase diffusion has been put forward, this degradation mechanism remains as yet unclear.[57] After approximately 18,000 hours of SOEC operation at $800^{\circ} \mathrm{C}$, the cells of an SOEC stack operated in Jülich show a strong depletion of $\mathrm{Ni}$ in the fuel electrode, as shown in Figure $10 \mathrm{a}$ ). For a more detailed analysis, refer to the post-test analysis of Frey and co-workers.[55] It is noteworthy that the overall degradation of the stack was dominated by an increase in the ohmic resistance, which was much more severe than the increase of the polarization resistance.[21]

In order to examine this issue, we apply the DRT analysis to one of the cells after 18,000 hours of SOEC operation. Figure $10 \mathrm{~b}$ ) shows the characteristic frequencies of the cell against reciprocal temperature, as determined from the DRT fit. The grain boundary contributions are shown as blue circles, and the reference frequencies of the dense YSZ ceramic is shown as a solid blue line. For comparison, the characteristic frequencies of the grain boundary contribution of the cell after long-term SOFC operation (discussed in 3.3.2) is shown as brown, hollow circles. We find that the frequencies for the YSZ grain boundaries after SOEC operation are distinctly lower than the reference frequencies, and essentially identical to the characteristic frequencies of the cell after long-term SOFC operation. This indicates the presence of $\mathrm{Mn}$-ions on the grain boundaries of both the SOEC and SOFC cell after a lengthy operation at elevated temperature, supporting the conclusion that the mechanism for this degradation is mainly chemical diffusion, since the opposite cell polarization has a negligible influence on this effect. This finding is supported by the post-test analysis using SEM-EDX.[55]

In contrast to the SOFC cell (open red squares), the characteristic frequency of the YSZ grain transport (solid red squares in Figure 10 b)) in the SOEC cell is also lower than that of the bulk reference (solid red line). While the characteristic frequency is related to the mobility of the charge carriers and can therefore be influenced by the doping concentration, a notable diffusion of $\mathrm{Y}^{3+}$ ions is unlikely at the operation temperature of $800^{\circ} \mathrm{C}$. However, changes in the frequency dispersion of the YSZ grain transport have been shown to be related to porosity.[58] The migration of metallic Ni away from the electrolyte in the fuel electrode shifts the electrochemically

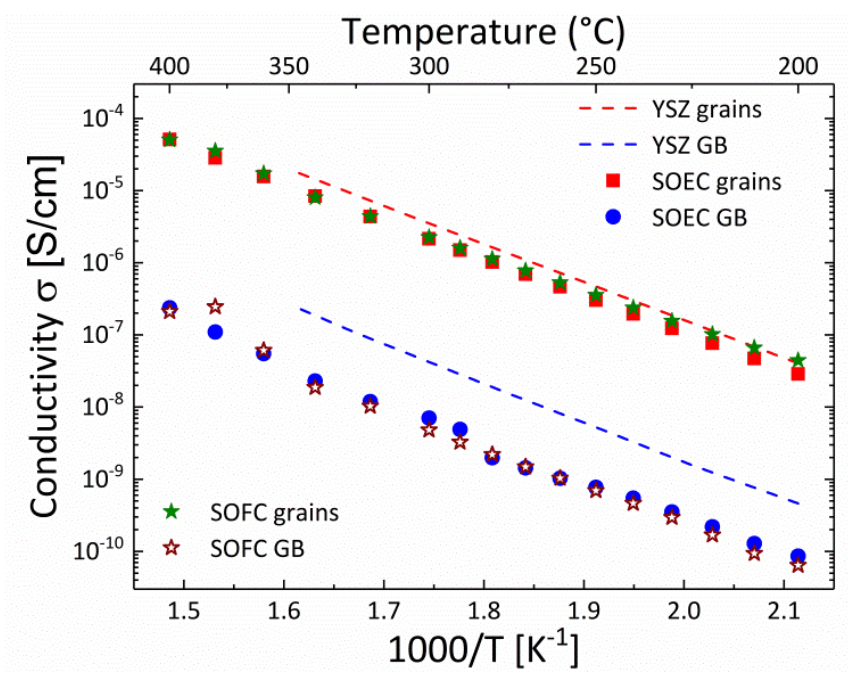

Figure 11: Calculated conductivity values for grain and grain boundaries vs. reciprocal temperature for the degraded SOEC cell (red squares and blue circles, respectively) and the degraded SOFC cell (solid, green stars and open, brown stars, respectively). The red and blue dashed lines represent the reference values for the bulk YSZ grain and grain boundary conductivity, respectively, of a dense ceramic. active zone continuously further away from the actual electrolyte, increasing the effective electrolyte thickness by a highly porous layer of YSZ. The porosity in the fuel electrode was determined as $\sim 45 \%$ in the area of the fuel inlet,[55] leading to a much higher area specific resistance compared to the electrolyte. Steil and coworkers determined that the conductivity of porous YSZ could be related to the bulk conductivity of YSZ via the relationship:[58]

$\sigma_{\text {bulk }}=\sigma_{\text {bulk }}^{0}[1-P]^{2.74}$ 
with $\sigma_{\text {bulk }}$ and $\sigma_{\text {bulk }}^{0}$ being the effective (measured) conductivity and the bulk conductivity of dense YSZ, respectively, and P the porosity. This formula predicts that a porosity of $45 \%$ results in a 5 -fold increase in ASR relative to the dense material. In a simple approach, the area specific ohmic resistance of the electrolyte and the degraded, porous anode can be described as a serial connection of two resistors, with $A S R_{\text {ohm }}=A S R_{\text {electrolyte }}+A S R_{\text {fuel electrode }}=\sigma_{Y S Z}^{-1}\left(l_{\text {Electrolyte }}+l_{\text {Anode }} *(1-P)^{2.74}\right)$, where $I$ is the thickness of each layer. The thickness of the anode layer, in this case, refers to the thickness of the layer from which Ni has migrated away completely, or where the degradation is sufficiently advanced to disrupt the percolated network of Ni grains which would otherwise provide an electrical short circuit of the porous anode. Using Figure 10 a) as a guide, the thickness of the depletion layer is approximately $5 \mu \mathrm{m}$ in the area of the fuel inlet.

Figure 11 shows the conductivity of grains and grain boundaries for the degraded SOEC and SOFC (see section 3.3.2) cells, calculated using the information about the layer thickness and porosity. For the grain boundary conductivity, the layer thickness used for calculation is the equal to the thickness of the dense electrolyte, while the grain conductivity is calculated using the sum of dense electrolyte and porous anode layer. Using this geometrical correction, the calculated ionic conductivity of the SOEC and SOFC cells are essentially identical. While the grain conductivity is very close to that expected from the bulk reference, the grain boundary conductivity is decreased due to Mn impurities. This calculation demonstrates that the increase in ohmic resistance during SOEC operation is entirely due to the increase in effective electrolyte thickness. From this finding, a possible mitigation for this type of degradation would be an increase of the ceramic content of the fuel electrode in order to decrease the porosity of the degraded layer.

\section{Conclusions}

We have demonstrated a novel technique to investigate the ionic conductivity of thin, supported ion conductors via a combination of impedance spectroscopy and fitting of DRT spectra. Using this technique, it is possible to examine the ionic conductivity of supported electrolytes in detail and free of the influence of parasitic ohmic resistances (such as contact resistance). Giving three examples relevant to SOFC and SOEC operation, we have shown that this technique can be applied to understand degradation effects in supported electrolytes affecting both grain and grain boundary conductivity. The ability to analyse the actual component after or during operation is indispensable to understand degradation effects caused by the cell fabrication or stack operation. We expect that the same analysis can be applied to other types of supported, ceramic membranes such as proton conductors, gas separation membranes and Li- or Na-conductors.

\section{Conflicts of interest}

There are no conflicts to declare.

\section{Acknowledgements}

The authors gratefully acknowledge all scientific and technical staff at Forschungszentrum Jülich that contribute towards stack testing and assembly, for providing the cells after the long-term SOFC and SOEC tests. Furthermore, the authors would like to thank A. Weber (from KIT, IAM-WET, Germany) and Q. Fang for fruitful discussions.

\section{Notes and references}

[1] B.-Y. Chang, S.-M. Park, Annual Review of Analytical Chemistry 3 (2010) (1) 207.

[2] N. Bonanos, B.C.H. Steele, E.P. Butler, Impedance Spectroscopy, John Wiley \& Sons, Inc. (2005), p.205-537.

[3] E. Ivers-Tiffée, A. Weber, H. Schichlein, Handbook of Fuel Cells, John Wiley \& Sons, Ltd (2010).

[4] B.A. Boukamp, Solid State lonics 169 (2004) (1-4) 65.

[5] R. Murugan, V. Thangadurai, W. Weppner, Angewandte Chemie International Edition 46 (2007) (41) 7778.

[6] J. Shim, R. Kostecki, T. Richardson, X. Song, K.A. Striebel, Journal of Power Sources 112 (2002) (1) 222.

[7] D. Andre, M. Meiler, K. Steiner, C. Wimmer, T. Soczka-Guth, D.U. Sauer, Journal of Power Sources 196 (2011) (12) 5334.

[8] S.B. Adler, J.A. Lane, B.C.H. Steele, Journal of The Electrochemical Society 143 (1996) (11) 3554.

[9] J. Jamnik, J. Maier, Physical Chemistry Chemical Physics 3 (2001) (9) 1668.

[10] F.S. Baumann, J. Fleig, H.-U. Habermeier, J. Maier, Solid State lonics 177 (2006) (11-12) 1071.

[11] A. Flura, C. Nicollet, S. Fourcade, V. Vibhu, A. Rougier, J.M. Bassat, J.C. Grenier, Electrochimica Acta 174 (2015) 1030.

[12] A. Leonide, V. Sonn, A. Weber, E. Ivers-Tiffée, Journal of The Electrochemical Society 155 (2008) (1) B36.

[13] A. Häffelin, J. Joos, M. Ender, A. Weber, E. Ivers-Tiffée, Journal of The Electrochemical Society 160 (2013) (8) F867.

[14] C. Endler-Schuck, J. Joos, C. Niedrig, A. Weber, E. Ivers-Tiffée, Solid State lonics 269 (2015) 67.

[15] C. Haering, A. Roosen, H. Schichl, M. Schnöller, Solid State lonics 176 (2005) (3) 261.

[16] M.R. Terner, J.A. Schuler, A. Mai, D. Penner, Solid State lonics 263 (2014) 180.

[17] F. Tietz, D. Sebold, A. Brisse, J. Schefold, Journal of Power Sources 223 (2013) 129.

[18] M.A. Laguna-Bercero, R. Campana, A. Larrea, J.A. Kilner, V.M. Orera, Journal of Power Sources 196 (2011) (21) 8942.

[19] J. Schefold, A. Brisse, H. Poepke, International Journal of Hydrogen Energy 42 (2017) (19) 13415. 
[20] Y. Tao, S.D. Ebbesen, M.B. Mogensen, Journal of Power Sources 328 (2016) 452.

[21] Q. Fang, C.E. Frey, N.H. Menzler, L. Blum, Journal of The Electrochemical Society 165 (2018) (2) F38.

[22] D. Kennouche, Q. Fang, L. Blum, D. Stolten, Journal of The Electrochemical Society 165 (2018) (9) F677.

[23] J.T.S. Irvine, D.C. Sinclair, A.R. West, Adv. Mater. 2 (1990) (3).

[24] M.C. Martin, M.L. Mecartney, Solid State lonics 161 (2003) (1) 67.

[25] W.K. Lee, J.F. Liu, A.S. Nowick, Physical Review Letters 67 (1991) (12) 1559.

[26] I.M. Hodge, M.D. Ingram, A.R. West, Journal of Electroanalytical Chemistry and Interfacial Electrochemistry 74 (1976) (2) 125.

[27] J. Bisquert, V. Halpern, F. Henn, The Journal of Chemical Physics 122 (2005) (15) 151101.

[28] C. Ahamer, A.K. Opitz, G.M. Rupp, J. Fleig, Journal of The Electrochemical Society 164 (2017) (7) F790.

[29] B.A. Boukamp, A. Rolle, Solid State lonics 314 (2018) 103.

[30] E. Ivers-Tiffée, A. Weber, Journal of the Ceramic Society of Japan 125 (2017) (4) 193.

[31] H. Schichlein, A.C. Müller, M. Voigts, A. Krügel, E. Ivers-Tiffée, Journal of Applied Electrochemistry 32 (2002) (8) 875.

[32] A.B. Tesler, D.R. Lewin, S. Baltianski, Y. Tsur, Journal of Electroceramics 24 (2010) (4) 245.

[33] M. Saccoccio, T.H. Wan, C. Chen, F. Ciucci, Electrochimica Acta 147 (2014) 470.

[34] T.H. Wan, M. Saccoccio, C. Chen, F. Ciucci, Electrochimica Acta 184 (2015) 483.

[35] S. Hershkovitz, S. Tomer, S. Baltianski, Y. Tsur, ECS Transactions 33 (2011) (40) 67.

[36] W. Schafbauer, N.H. Menzler, H.P. Buchkremer, International Journal of Applied Ceramic Technology 11 (2014) (1) 125.

[37] S. Uhlenbruck, N. Jordan, D. Sebold, H.P. Buchkremer, V.A.C. Haanappel, D. Stöver, Thin Solid Films 515 (2007) (7) 4053.

[38] B.A. Boukamp, Journal of The Electrochemical Society 142 (1995) (6) 1885.

[39] M. Schönleber, D. Klotz, E. Ivers-Tiffée, Electrochimica Acta 131 (2014) 20.

[40] M. Schoenleber, E. Ivers-Tiffée, Meeting Abstracts MA2016-02 (2016) (23) 1687.

[41] W. Rasband, https://imagej.nih.gov/ij/index.html.

[42] M. Ettler, H. Timmermann, J. Malzbender, A. Weber, N.H. Menzler, Journal of Power Sources 195 (2010) (17) 5452

[43] T. Van Gestel, D. Sebold, H.P. Buchkremer, D. Stöver, Journal of the European Ceramic Society 32 (2012) (1) 9.

[44] F. Han, R. Mücke, T. Van Gestel, A. Leonide, N.H. Menzler, H.P. Buchkremer, D. Stöver, Journal of Power Sources 218 (2012) 157.

[45] E.-C. Shin, P.-A. Ahn, J.-M. Jo, H.-S. Noh, J. Hwang, J.-H. Lee, J.-W. Son, J.-S. Lee, J. Korean Ceram. Soc 49 (2012) (5) 404.

[46] T.M. Huber, A.K. Opitz, M. Kubicek, H. Hutter, J. Fleig, Solid State Ionics 268 (2014) 82.

[47] L. Zhang, S.P. Jiang, H.Q. He, X. Chen, J. Ma, X.C. Song, International Journal of Hydrogen Energy 35 (2010) (22) 12359.

[48] M. Gerstl, A. Nenning, R. Iskandar, V. Rojek-Wöckner, M. Bram, H. Hutter, A. Opitz, Materials 9 (2016) (8) 649.

[49] C. Lenser, H. Jeong, Y.J. Sohn, N. Russner, O. Guillon, N.H. Menzler, Journal of the American Ceramic Society 101 (2017) (2) 739.

[50] A. Tsoga, A. Naoumidis, D. Stöver, Solid State lonics 135 (2000) (1-4) 403.

[51] V. Rührup, H.D. Wiemhöfer, Z. Naturforschung 61b (2006) 916.

[52] S. Rodewald, J. Fleig, J. Maier, Journal of the American Ceramic Society 84 (2001) (3) 521.

[53] R. Hagenbeck, R. Waser, Journal of Applied Physics 83 (1998) (4) 2083.

[54] N.H. Menzler, D. Sebold, O. Guillon, Journal of Power Sources 374 (2018) 69.

[55] C.E. Frey, Q. Fang, D. Sebold, L. Blum, N.H. Menzler, Journal of The Electrochemical Society 165 (2018) (5) F357.

[56] K.V. Hansen, M. Chen, T. Jacobsen, K. Thydén, S.B. Simonsen, S. Koch, M.B. Mogensen, Journal of The Electrochemical Society 163

(2016) (10) F1217.

[57] M.B. Mogensen, A. Hauch, X. Sun, M. Chen, Y. Tao, S.D. Ebbesen, K.V. Hansen, P.V. Hendriksen, Fuel Cells 17 (2017) (4) 434.

[58] M.C. Steil, F. Thevenot, M. Kleitz, Journal of The Electrochemical Society 144 (1997) (1) 390. 